\title{
Wave Propagation Characterization in Complex Urban Areas using EMTerrano
}

\author{
F Aryanfar ${ }^{1 *}$ Student Member, IEEE, K. Sarabandi ${ }^{1}$ Fellow, IEEE \\ M. D. Casciato ${ }^{2}$ Member, IEEE, and K. Sabet ${ }^{2}$ Member, IEEE \\ 1 The University of Michigan, 1301 Beal Avenue, Ann Arbor, MI, 48109-2122 \\ Phone:(734) 763-2153, Email: faryanfa@engin.umich.edu \\ 2 EMAG Technologies, 1340 Eisenhower Place, Ann Arbor, MI, 48108 \\ Phone:(734) 973-6600, Email: casciato@eecs.umich.edu
}

\section{INTRODUCTION}

The trend in propagation prediction in recent years has been toward the development of physics-based propagation models [1], [2], which represent various features in the physical environment and tend to be more accurate than heuristic or simplified analytic models. These models are site-specific, and the intent is to allow for the simulation of any environment for which the physical scenario is know.

Recently, the trend in physics-based propagation prediction for urban areas has been toward the so called quasi-3D methods, such as the vertical plane launch method (VPL) [3]. Their application is based on the premise that the computational time required for full $3-\mathrm{D}$ ray-tracing (covering $4 \pi$ steradians) in a highly scattering environment is prohibitive. Based on this premise, methods such as VPL, confine the ray coverage area to the 2$\mathrm{D}$, horizontal plane, and extend the rays to the 3-D plane, by calculating the difference between transmitter and receiver heights. Ground reflection is accounted for by applying image theory. These methods however, apply only 2-D reflection and diffraction coefficients, which, at each ray intersection point, can accumulate significant error in the received ray strength. It is expected that this error will be especially significant for situations in which the transmitter and receiver are at a substantial height difference. In addition, for indoor scenarios, a full 3D ray tracer is essential to account for both floor and ceilings.

Because of these limitations in the quasi-3D approaches, an investigation has begun into methods for improving the computational efficiency of full 3D ray tracing, so as to make it of practical use in propagation prediction. With this in mind, a full 3D ray tracing algorithm [4] has been incorporated into the propagation simulation tool EMTerrano, developed at EMAG Technologies, Ann Arbor, Michigan. By the application of "intelligent ray tracing," preliminary results are shown in this paper, which testify as to the practicality of using a full $3 \mathrm{D}$ ray tracing engine in a propagation simulation tool such as EMTerrano.

\section{INTELLIGENT 3D RAY TRACING}

The basis for intelligent 3D ray tracing in EMTerrano is to apply a simple, common sense approach to ray tracing in three dimensions. The following basic assumptions are made:

1) The ray generated from each source point (source, reflection, diffraction) will not interact with any object behind, or to the sides of it.

2) When checking for ray interaction with a given receiver, the same assumption can be made.

3) The sole criteria for when to discard a particular ray is it's power amplitude, based on receiver sensitivity, not the number of reflections or diffractions that it has gone through.

Figure $1 a$ illustrates the concept described in item 1 above. Basically the user defines an angular search area, in steradians, for each ray generated by the transmitter, reflected by a building (or the ground), or diffracted by a corner. For each one of these ray sources, only objects in the angular area defined are checked for interception by the given ray. Application of this algorithm can result in significant savings in time, proportional to the reduction in the angular search area for each ray, without any significant degradation in 
accuracy. Applying the same concept to test for rays captured by a receiver, a further $30 \%$ reduction in simulation run time is observed. While further investigation must be performed, it is believed that item 3 above will result in additional improvements in computer run times, especially in more densely populated scattering environments. Many ray tracers base the decision on when to disregard a ray, at least in part, on the number of reflections or diffractions that a ray undergoes. In a highly scattering environment, this number must be set fairly high, as it is more likely that the dominate ray path will be one with significant reflections. This is inefficient however, as many rays, whose power levels have dropped below the receiver sensitivity, will continue to be traced. Basing the criteria for disregarding a ray solely on it's power level (usually set to the expected receiver sensitivity), will result in maximum efficiency in the ray tracing algorithm, both in terms of accuracy and simulation run time.

\section{ACcuracy OF QUaSI-3D Ray Tracing}

As mentioned previously, quasi-3D ray methods usually apply $2 \mathrm{D}$ reflection and diffraction coefficients, that is, it is assumed that the ray is always incident in a plane perpendicular to the vertical in the propagation scenario. Depending on the actual incident angle of the $3 \mathrm{D}$ ray with the building or object structure, significant error in the reflection or diffraction coefficients can be introduced. This error increases as the actual incident angle of the ray moves away from the 2D plane, and at or near Brewster's angle (parallel polarization only), where no reflected field is present, can become even more acute. The effects of this error are cumulative, increased at each interaction point for a given ray. To illustrate this point Figures $1 b$ and $c$ show the percent error (in $\mathrm{dB}$ ) in the magnitudes of the $2 \mathrm{D}$ Fresnel reflection coefficients applied in the quasi-3D methods, when compared to the magnitudes of the actual 3D reflection coefficients, as a function of the 3D incident angles $\theta_{3 D}$ and $\phi_{3 D}$. Figure $1 b$ shows the percent error for perpendicular polarization and Figure $1 c$ for parallel polarization, where the percent error $E$, is defined as, $E=\left(\left|\Gamma_{3 D}\right|-\left|\Gamma_{2 D}\right|\right) /\left|\Gamma_{3 D}\right|$, and the angle used in the calculation of the approximate $2 \mathrm{D}$ reflection coefficients, $\theta_{2 D}$, is determined from the 3D angles by, $\theta_{2 D}=\arctan \left(\left|k_{x}\right| / \cos \theta_{3 D} / k_{0}\right)$, where $k_{0}$ is the free space propagation constant, and $\left|k_{x}\right|$ is the magnitude of the $x$ component of $k_{0}$, given by, $\left|k_{x}\right|=\left|k_{0} \sin \theta_{3 D} \cos \phi_{3 D}\right|$. Figure $1 d$ shows the geometry of the problem.

\section{RESULTS}

In this section some example results will be shown from the $3 \mathrm{D}$ ray tracer, and compared with those generated from a VPL engine, also available in EMTerrano. The 3D engine directly includes all ground reflections, while the VPL, using the method of images (this VPL assumes the same path length for direct and reflected field amplitudes), only includes the ground reflection between the transmitter and first obstacle, and between the last obstacle and the receiver. Also note that building penetration, available in the $3 \mathrm{D}$ engine, is turned off, for a reasonable comparison between the two methods. Receiver sensitivity was set for the 3D method at $-90 \mathrm{dBm}$, relative to the transmitted signal power of $1 \mathrm{~W}(120 \mathrm{~dB}$ range of path loss), while in the VPL simulation the number of reflections is limited to 10. Figure $2 a$ shows a rendition of downtown Ann Arbor, Michigan, which will be used as the example scenario. It consists of 84 buildings of various heights (note that while the VPL engine accounts for over rooftop diffraction, the $3 \mathrm{D}$ ray tracer currently does not). A vertical transmitter, operating at $1 \mathrm{GHz}$, is located at Point $\mathrm{A}$ in the figure (Tx), and placed at $12 \mathrm{~m}$ height. To determine coverage in the scenario, a grid of 5700 vertically polarized receivers is placed throughout the scene, at $2 \mathrm{~m}$ height. The permittivity of both the ground and all buildings are assumed to be $\epsilon_{r}=6.0$, and the conductivity of both is $\sigma=0.005 \mathrm{~S} / \mathrm{m}$.

Figure $2 b$ shows the path loss (in $\mathrm{dB}$ ), for the scenario, simulated using the VPL engine, while Figure $2 c$ shows results from the full 3D ray tracer. The differences in the two sets of results is best observed in Figure $2 d$, which shows the absolute difference between the two methods. While the positive difference in levels can to some extent be attributed to the inclusion of rooftop diffraction in the VPL calculations (the difference, $\triangle$ Path Loss in 


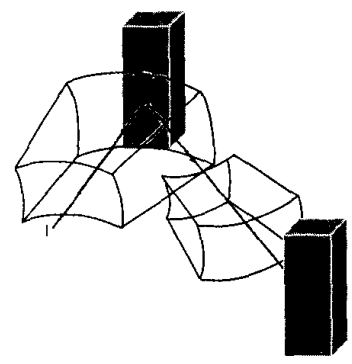

(a)

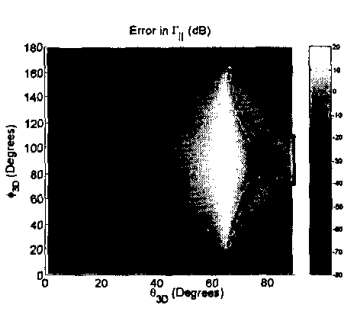

(c)

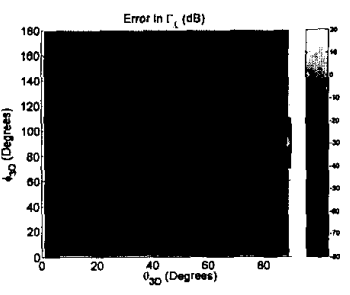

(b)

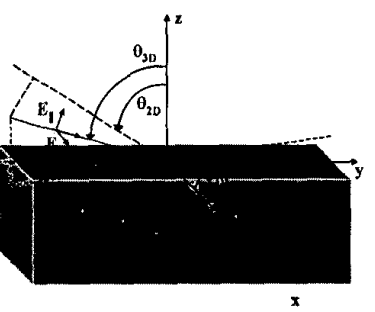

(d)

Fig. 1. Intelligent ray tracing (a), Error in Fresnel Reflection Coefficients for $2 \mathrm{D}$ approximation, (b) Perpendicular Polarization, (c) Parallel Polarization, (d) problem geometry.

the figure, is defined as the path loss levels of the VPL minus the path loss levels of the $3 \mathrm{D}$ engine), the large negative values in Figure $2 d$ can be attributed to the inclusion of full $3 \mathrm{D}$ effects in the 3D ray tracer. For example, note the darker areas in the building shadows at width (vertical axis in the figure) equaling 0 to $50 \mathrm{~m}$, and at length (horizonta axis in the figures) equaling 0 to $50 \mathrm{~m}, 150$ to $200 \mathrm{~m}$ and approximately 225 to $325 \mathrm{~m}$, which shows a difference of up to at least $-20 \mathrm{~dB}$. As an example of the efficiency of the intelligent ray tracing method described previously, a timing comparison is given in Table I. The table shows runtimes for the 3D engine, with building penetration both on and off, and for the VPL with and without rooftop diffraction. Simulations were done on a $\mathrm{PC}$, with a 1.7 GHz processor, and with $500 \mathrm{MB}$ of RAM. The angular search area set for the 3D engine simulations was $\pi / 2$ steradians. Considering that the VPL is in fact searching $2 \pi$ steradians (remember in the quasi-3D methods, rays are launched $360^{\circ}$ in the horizontal plane only) the ratio of the search area for the VPL method to that of the 3D engine is 4, therefore a speed-up in the runtime for the 3D method, over that of the VPL of approximately 4 would be expected, but as noted in the table, the speed-up is in fact greater than 4 . The difference may be attributed to other factors, for example the efficiency of the implemented algorithms in both engines. 


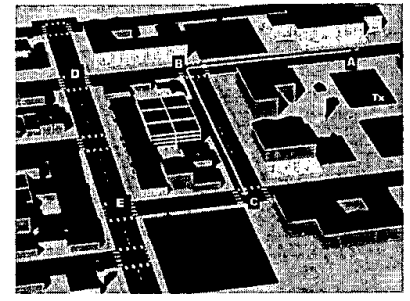

(a)

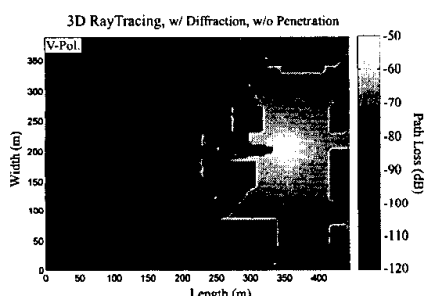

(c)

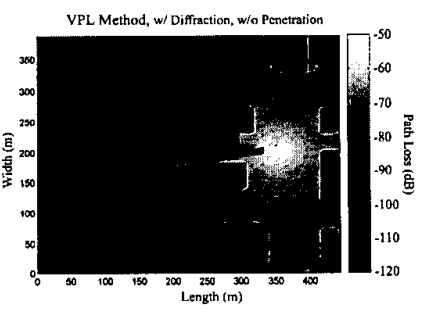

(b)

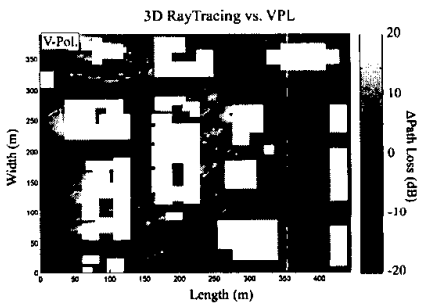

(d)

Fig. 2. Comparison of the quasi-3D VPL method with full 3D ray tracing for downtown Ann Arbor, Michigan. (a) Rendition of Ann Arbor, Michigan, (b) VPL results, (c) 3D results, (d) VPL path loss minus $3 \mathrm{D}$ path loss.

$$
\text { TABLE I }
$$

ANN ARBor, Michigan: Run tIME COMPARISON FOR VPL, AND 3D (84 BUILdingS, 5700 RECEIVERs)

\begin{tabular}{|c|c|c|c|c|c|}
\hline Method & Rooftop Diffraction & Reflection & Diffraction & Transmission & Run time (min.) \\
\hline 3D & no & yes & yes & no & 21 \\
3D & no & yes & yes & yes & 27 \\
VPL $* *$ & no & yes & yes & no & 110 \\
VPL & yes & yes & yes & no & 120 \\
\hline
\end{tabular}

\section{ACKNOWLEDGMENT}

The authors would like to thank the Defense Advanced Research Projects Agency (DARPA) for their support for this work under contract number DAAH01-03-C-R116 and Daniel Mendez and Saivilasini Niranjan.

\section{REFERENCES}

[1] I. Koh, F Wang and K. Sarabandi, "Estimation of coherent field attenuation through dense foliage including multiple scattering," IEEE Trans. on Geoscience and Remote Sensing, vol. 41, no. 5, May

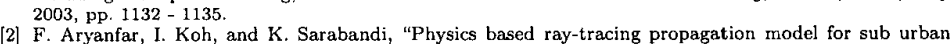
areas," Proc. of the AP-S Int. Symp., vol. 4, June 2003, pp. $903-906$

. A Bertoni "A new approach to 3-D ray tracing for propagation prediction in cities," IEEE Trans, on Ants. and Prop., vol, 46, no. 6, June 1998, pp. $853-863$.

[4] F. Aryanfar and S. Safavi-Naeini "Electromagnetic modeling of radio-wave propagation in micro- and pico-cellular environments, "IEEE Antennas and Propag. For Wireless Communication Conf., November 1998, pp. 2528 